\title{
Introduction to Raman Microscopy/ Spectroscopy
}

\author{
Dejan Lazić
}

\begin{abstract}
Raman microscopy/spectroscopy is a non-destructive technique that affords detection of vibrational, rotational or other low frequency excitations of the observed system. Vibration will be active in the Raman spectrum when it causes a change in polarizability of molecule. Characteristics of Raman spectra depend on the mass of the atoms, their geometrical arrangement in the molecule, as well as the intensity of the forces that connect these atoms. Coupling Raman spectroscopy with microscopy afforded obtaining precise morphologi$\mathrm{cal}$ and structural images of the observed sample. A large number of papers has been published in the field of physico-chemical characterization of materials using Raman microspectroscopy. Its use has slowly propagated into biological sciences as a method for quality control of pharmaceutical products. Application of Raman microspectroscopy will expand as researchers of biological systems become more aware of the possibilities of this method.
\end{abstract}

Spectroscopy is the field that deals with the study of radiation after its interaction with matter. Such radiation may have electromagnetic or mechanical properties or it might be composed of particles. In order for frequency spectrum

How to cite this book chapter:

Lazić, D. 2019. Introduction to Raman Microscopy/Spectroscopy. In: Vucelić Radović, B., Lazić, D. and Nikšić, M. (eds.) Application of Molecular Methods and Raman Microscopy/Spectroscopy in Agricultural Sciences and Food Technology, Pp. 143-150. London: Ubiquity Press. DOI: https://doi.org/10.5334/bbj.i. License: CC-BY 4.0 
of electromagnetic radiation to be used, interval of the frequencies can be between frequencies of radio waves to the frequency of $\gamma$ radiation, whereas for the radiation made up from particles interaction with electrons, neutrons, muons, and in some cases the entire atoms or ions can be used. Information on the matter is obtained from the spectrum that occurs after interaction of radiation with the matter (Kuzmany 2009).

Due to the possibility of determining physical and chemical properties of a substance, Raman microscopy is used in chemistry, medicine, astronomy, metallurgy.

The methods used in spectroscopy vary depending on which substance is used, an atom, molecule or substance, and of the properties of matter that are being tested (http://web.mit.edu/spectroscopy/history/history-classical.html). Thus, spectroscopy as a discipline can be divided into several sub-disciplines, according to:

- The type of radiation - it'sfrequency (radio, microwave, and ahercna, infrared, visible, ultraviolet, X-ray, gamma)

- Level of interaction (nuclear, atomic, molecular, aggregation)

- Inventor (Raman, Mesbauerova)

- Technique (Fourier, resonance, laser, coherent, emission, absorbent)

Based on the phenomenon that causes coupling of electromagnetic radiation spectroscopy can be divided into:

- rotational spectroscopy

- electron spectroscopy

- vibrational spectroscopy

(Http://web.mit.edu/spectroscopy/history/history-classical.html)

Vibrational spectroscopy is the branch of spectroscopy that studies the spectra, which are caused by vibrations of molecules. This includes infrared spectroscopy and Raman spectroscopy. It is used for identification of molecules based on their atomic vibrations, and periodic changes in the angles and distances of atoms.

Vibrational spectroscopic techniques can be based on the absorption or dispersion of electromagnetic radiation, and therefore we can distinguish between infrared and Raman spectroscopy.

All molecules vibrate. In contrast to the vibrations of macroscopic bodies vibrations of molecules are quantum. Molecules can vibrate with very specific amplitudes which correspond to the vibrational energy levels. The spectroscopic transition can occur only if the energy of absorbed or emitted photon is equal to the difference between two vibrational energy levels (Bohr condition) or if the difference between energy of scattered and the energy of the incoming photon equals to the energy difference between levels (Figure 1). If vibrations 
of molecule cause change in the dipole moment of the molecule, coupling between such vibrations with vibrations of electromagnetic radiation will happen which causes either absorption or emission of radiation. Such vibrations will be active in the infrared spectrum (Crouch 2007). Vibration will be active in the Raman spectrum when it causes a change in polarizability of molecule.

The study of the spectrum of electromagnetic radiation is carried out using a spectroscope i.e. spectrometer. The analysis provides data on the presence of different types of atoms and molecules in matter.

Since the molecule represents a system of atoms which are linked together by certain forces, as a result of this resilient connection it is possible for the atoms within the molecule to oscillate. Oscillation of atoms and the changes occurring as a result of these oscillations are described by vibrational spectra. These characteristics depend on the mass of the atoms, their geometrical arrangement in the molecule, as well as the intensity of the forces that connect these atoms. Substances containing a large number of molecules such as crystals act like super molecules and in them individual oscillations of molecules can couple and intereact with each other. The exact explanation of these phenomena is not possible; therefore, certain approximations must be introduced. One of the introduced approximations is neglecting mutual interactions of molecules. As any substance or molecule has its own unique characteristics their vibrational spectra will vary. Using Raman spectroscopy one can identify the type of unknown molecule. Also, it is possible to determine molecular structure and to calculate other characteristics of the molecules from their vibrational spectra. That is why today this kind of spectroscopy plays an important role in science.

\section{Raman spectroscopy}

Sir Chandrasekhara Venkata Raman (1888 -1970) was an Indian physicist whose work largely influenced the development of physics. In 1930 he won the Nobel Prize for Physics for explaining the effect of light changing its wavelengths as it passed through the transparent material (Raman scattering) (http://en.wikipedia.org/wiki/C._V._Raman).

Inelastic reflections of light in interaction with matter theoretically predicted Brillouin in 1922. The first to observe it in experiments were Raman and Krishnan in 1928. For the light source they used focused and filtered beam of sunlight, the sample was liquid in the bucket, a detector was an eye. The same principle applies today. During development of the method as a light source different lamps, lasers were used and today laser diodes prevail, while photomultipliers or CCD cameras are used as the detectors.

Raman spectroscopy uses inelastic scattering of monochromatic light (usually a laser). Raman scattering is used to gather spectroscopic data. Inelastic scattering means that the frequency of monochromatic photons in the light changes after its interaction with the sample. The electromagnetic radiation 
that is scattered by a molecule contains two components which come from the vibration or the rotation of the molecules (Schrader 1995). Photon that exits the system does not have the same energy as a photon entering the system. Raman scattering system receives or loses power. The difference in the energy of the system before and after scattering corresponds to the difference between the energy of the incoming and outgoing photons. If the outgoing photon has lower energy of incoming photons then the system will recieve energy through scattering. This type of scattering is called Stokes scattering, in the opposite case, the scattering is called anti-Stokes (Figure 1).

Energy of spectroscopic transfer is determined by the energy difference of Stokes or anti-Stokes band and the wavelength of incident monochromatic radiation. As the Stokes and anti-Stokes bands are of much lower intensity than scattered Rayleigh ${ }^{\star}$ radiation it is necessary to apply a high-intensity radiation source (Schrader 1995).

${ }^{*}$ Rayleigh scattering in the atmosphere is due to the scattering of light on air molecules (whose dimensions are much smaller than the wavelength of the incident radiation) that are moving chaotically, thus it is the result of the fluctuations in air density (leading to different values of refractive index in the areas of different densities).

Stokes and anti-Stokes scattering depends on the change in polarizability of molecules in time and in the Raman spectrum only the vibrations and rotations that modify the polarizability of molecules can be observed. Therefore, in the Raman and infrared spectra, the same bands usually have different intensities, and sometimes they are in one of these spectra completely invisible. Therefore, infrared and Raman spectroscopy are considered complementary (Schrader 1995)

Raman spectroscopy has a great advantage over infrared spectroscopy because Raman spectra can be recorded in aqueous solutions. Raman spectroscopy can be also used for the study of solid and gas samples. Around 99.999\% of all photons in spontaneous Raman spectroscopy belong to elastic Rayleigh scattering category. This type of scattering is useless for practical application for molecular characterization. Only $0.001 \%$ of the incident monochromatic light produces inelastic Raman signal of frequency Vo \pm $\mathrm{Vm}$. Spontaneous Raman scattering is very weak and it is necessary to take specific measures in order to differentiate it from the prevailing Rayleigh scattering.

Raman spectroscopy system typically consists of four main components: the source of radiation that is usually a continuous laser type system for illuminating the sample, the system for separating wavelengths and the systems for detection and signal processing (Ferraro 2002).

Raman spectrophotometers use the source of monochromatic radiation that is directed at the sample. Radiation, scattered at an angle (usually $90^{\circ}$ ) is leaded to the monochromator which allows radiation of only one wavelength to go 
out. Spectrum is obtained by scanning in the wavelengths around the wavelength of the radiation source.

Raman spectra can be:

- rotational

- rotaional vibrational and

- electron (rarely)

Raman effect is based on inelastic scattering of monochromatic incident radiation (laser radiation) from a ultraviolet, visible or near IR part of spectra.

- When light passes through a transparent material, the largest part of the light is scattered in all directions.

- Majority of the light, consisting of photons whose energy is not converted, is known as Rayleigh scattering.

- The much smaller part of the incident radiation having a change in energy is known as Raman scattering.

- The difference in energy is directly dependent on the molecular species present in the assay system.

If the incident radiation has a discrete spectrum then the spectrum of the scattered radiation in the three components occurs:

- radiation of the same frequency as the incident radiation (Rayleigh scattering)

- radiation of a higher frequency of the incident radiation (Stokes ANTI-RADIATION) and

- radiation of lower frequency of the incident radiation (Stokes RADIATION)

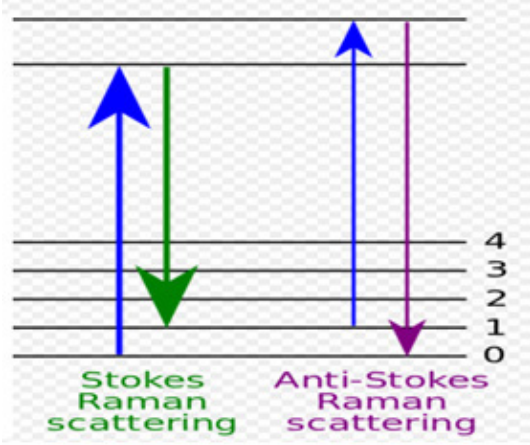

virtual states

excited

vibrational states

ground

Figure 1: Stokes and anti-Stokess Raman scattering. 


\subsection{Surface-enhanced Raman spectroscopy (SERS) and surface- enhanced resonance spectroscopy}

Spectroscopy of surface enhanced Raman scattering (SERS) is a method that has been developing in the last thirty years, and owing to its very high sensitivity it found application in numerous areas such as chemical and biochemical analysis, medicine, forensics and archeology (McNay et al. 2011, Schlüker et al. 2011). SERS spectroscopy provides vibrational spectrum of the molecule that has been adsorbed onto metal nanostructured surface. Practical use of SERS is difficult due to the formation of spectrum that is very difficult to interpret. It is extremely necessary to take into account physical and chemical factors influencing appearance and intensity of lines in the spectrum. To overcome these complications a type of SERS that uses amplification effect due to adsorption on the surface and Raman resonance was developed. It gives signal intensity up to even $10^{14}$ (Stiles et al 2008; Schatz and Van Duyne, 2002). The most important reason for the development of SERS technique is its applicability in various fields of natural sciences. By increasing intensity of scattered radiation on a metal substrate molecule, sensitivity of Raman spectroscopy is also increased and allows structural analysis of molecules based on vibrational spectrum. Since spectroscopy of surface enhanced Raman scattering has exceptional sensitivity and provides information on the structure of molecules, it is an ideal technique for examination of various chemical and biological systems. An additional advantage of the SERS spectroscopy is fluorescence quenching near the metal surface due to transfer of energy from the molecule to the metal (Schlüker 2011).

\subsection{Raman microspectroscopy}

Coupling Raman spectroscopy with microscopy has become significant during 70-ies. In addition to its classical use, use of the electron beam in microscopy gave precise morphological and structural images of the observed sample. Scanning electron microscopy, $\mathrm{x}$-rey microanalysis and analytical electron microscopy give precise details of the elemental composition of the tested substance but these methods don't give information on structural coordination as can be obtained by Raman microspectroscopy. A large number of papers have been published in the field of physico-chemical characterization of materials using Raman microspectroscopy. Its use has slowly propagated into biological sciences as a method for quality control of pharmaceutical products. Application of Raman microspectroscopy will expand as researchers of biological systems become more aware of the possibilities of this method.

Although Raman microspectroscopy is a relatively new method, its popularity due to simple procedure of sample preparation and high number of data available from one spectrum has lead to development of a high number 
of specific protocols. Majority of protocols is focused on enhancing specifity and sensitivity of the method. This is done by modifying sample preparation, changing the parameters etc. (Butler et al. 2016). In order to facilitate data manipulation and to allow classification of samples based on their "spectral fingerprint“, new softwares and subprograms in already existing programs were developed (Table 1).

\begin{tabular}{|c|c|c|}
\hline Software & Website & Licence \\
\hline CytoSpec & http://www.cytospec.com/ftir.php & Comercial \\
\hline ImageLab & $\begin{array}{l}\text { http://www.imagelab.at/en_home. } \\
\text { html }\end{array}$ & Comercial \\
\hline $\begin{array}{l}\text { Biodata Toolbox } \\
\text { (in MATLAB) }\end{array}$ & $\begin{array}{l}\text { http://www.mathworks.com/ } \\
\text { matlabcentral/fileexchange/22068- } \\
\text { biodata-toolbox }\end{array}$ & Open acess \\
\hline $\begin{array}{l}\text { Extended Multiplicative Signal } \\
\text { Correction (EMSC) Toolbox }\end{array}$ & $\begin{array}{l}\text { http://www.models.life.ku.dk/ } \\
\text { emsctoolbox }\end{array}$ & Open acess \\
\hline IrootLab & https://code.google.com/p/irootlab/ & Open acess \\
\hline $\begin{array}{l}\text { Multivariate Image Analysis } \\
\text { (MIA) Toolbox }\end{array}$ & $\begin{array}{l}\text { http://www.eigenvector.com/ } \\
\text { software/mia_toolbox.htm }\end{array}$ & Open acess \\
\hline $\begin{array}{l}\text { Multivariate Curve } \\
\text { Resolution- Alternating Least } \\
\text { Squares (MCR-ALS) Toolbox }\end{array}$ & $\begin{array}{l}\text { http://www.cid.csic.es/homes/ } \\
\text { rtaqam/tmp/WEB_MCR/welcome. } \\
\text { htm }\end{array}$ & Comercial \\
\hline PLS Toolbox (in MATLAB) & $\begin{array}{l}\text { http://www.eigenvector.com/ } \\
\text { software/pls_toolbox.htm }\end{array}$ & Open acess \\
\hline $\begin{array}{l}\text { Raman Processing Program } \\
\text { (in MATLAB-a) }\end{array}$ & http://cares.wayne.edu/rp/ & Comercial \\
\hline Origin for Spectroscopy & $\begin{array}{l}\text { http://www.originlab.com/index. } \\
\text { aspx?go=Solutions/Applications/ } \\
\text { Spectroscopy }\end{array}$ & Comercial \\
\hline PeakFit & $\begin{array}{l}\text { https://systatsoftware.com/products/ } \\
\text { peakfit/ }\end{array}$ & Comercial \\
\hline $\begin{array}{l}\text { PyChem (in Python } \\
\text { programe) }\end{array}$ & http://pychem.sourceforge.net/ & Open acess \\
\hline PyVib2(in Python programe) & http://pyvib2.sourceforge.net/ & Open acess \\
\hline HyperSpec (in R programe) & $\begin{array}{l}\text { http://hyperspec.r-forge.r-project. } \\
\text { org/ }\end{array}$ & Open acess \\
\hline $\begin{array}{l}\text { The Unscrambler X (in R } \\
\text { programe) }\end{array}$ & http://www.camo.com/ & Comercial \\
\hline
\end{tabular}

Table 1: Softwares for data manipulation and classification of Raman spectra (adopted from Butler et al. 2016). 


\section{Acknowledgments}

Thanks to the effort of a group of professors of Faculty of Agriculture in Belgrade and with the support of the EU, with funds received through AREA project Raman microscope was purchased and is used for research in agriculture. Researchers from our Faculty had the opportunity to familiarize themselves with the method through trainings at the Institute of Physical Chemistry of the University of Jena (Germany), where they gathered top researchers in the field of application of Raman spectroscopy in biological systems. Here are presented their experiences, impressions, messages to future researchers in this field.

\section{References}

Butler H.J., Ashton L., Bird B., Cinque G., Curtis K., Dorney J., Esmonde-White K., Fullwood N.J., Gardner B., Martin-Hirsch P.L., Walsh M.J., McAinsh M.R., Stone N. \& Martin F.L. (2016). Using Raman spectroscopy to characterize biological materials. Nature Protocols, 11(4), 664-687. DOI: https:// doi.org/10.1038/nprot.2016.036

Kuzmany, H. (2009). Solid-State Spectroscopy. 2nd edn. Springer-Verlag, Berlin. DOI: https://doi.org/10.1007/978-3-642-01479-6

MIT Spectroscopy: The Era of Classical Spectroscopy. Retrieved from http:// web.mit.edu/spectroscopy/history/history-classical.html

Crouch, A. (2007) Principles of instrumental analysis. Thomson Brooks/Cole, Australlia. DOI: https://doi.org/10.1021/ed069pA224.1

Schrader, B. (1995). Infrared and Raman Spectroscopy. VCH, New York. DOI: https://doi.org/10.1002/9783527615438

C. V. Raman. Retrieved from http://en.wikipedia.org/wiki/C._V._Raman

Ferraro, J. R. (2002) Introductory Raman Spectroscopy. 2nd edtion. Academic Press. New York. ISBN: 9780122541056

McNay G., Eustace D., Smith W. E., Faulds K. \& Graham, D. (2011). Surfaceenhanced raman scattering (SERS) and surface-enhanced resonance raman scattering (SERRS): a review of applications. Applied Spectroscopy, 65, 825-837. DOI: https://doi.org/10.1366/11-06365.

Schlüker, S. (2011). Surface Enhanced Raman Spectroscopy: Analytical, Biophysical and Life Science Applications. Wiley-VCH. Weinheim. DOI: https://doi.org/10.1002/9783527632756

Stiles, P-L., Dieringer, J. A., Shah, N. C. \& Van Duyne, R. P. (2008) Surfaceenhanced Raman spectroscopy. Annual Review of Analytical Chemistry, 601-626. DOI: https://doi.org/10.1146/annurev.anchem.1.031207.112814

Shatz, G. C. \& Van Duyne, R. P. (2002). Electromagnetic Mechanism of Surface-enhanced Spectroscopy. In J. M. Chalmers \& P. R. Griffiths (Eds.), Handbook of Vibrational Spectroscopy (pp. 759-774), John Wiley \& Sons. Chichester. DOI https://doi.org/10.1007/3-540-33567-6_2 